\title{
Secuencia de formas deposicionales submarinas en la fractura del Canal Messier, Patagonia Central
}

\author{
José F. Araya-Vergara \\ Departamento de Geografía, Universidad de Chile \\ Casilla 3387 \\ Santiago, Chile
}

Recibido 26 agosto 1998; versión corregida 6 mayo 1999; aceptado 16 julio 1999.

RESUMEN. Se analiza la secuencia y emplazamiento de las formas deposicionales del fondo, para determinar fases de la morfogénesis. Mediante registros de ecosonda de alta resolución, se estableció la distribución de las morfoestructuras en el perfil longitudinal y por orden de antigüedad decreciente.

A lo largo del Canal Messier aparecen dos tipos de zonas submarinas: a) En ausencia de fiordos confluentes, los fondos son someros (-50 a $-450 \mathrm{~m})$; las formas de diamicton masivo están interestratificadas con capas de explanadas de represamiento en las cubetas más deprimidas y predominan en los espacios más someros; la referencia estratigráfica fundamental es un manto de sedimentos que sobreyace a todo el conjunto, como fenómeno regional; b) En presencia de fiordos confluentes, la excavación del fondo es profunda $(-50$ a $-1350 \mathrm{~m})$; las formas de diamicton masivo interestratificadas con explanadas de represamiento son mucho más desarrolladas y similares a las de la zona externa de los fiordos; como referencia estratigráfica, falta el manto de cobertura.

Consecuentemente, el manto de cobertura debe haberse instalado cuando el hielo aún permanecía frente a cada fiordo, pero había desaparecido en los sectores de aguas someras. Estos rasgos parecen estar relacionados con incidencias de rápido retroceso glacial detectadas en investigaciones anteriores.

Palabras claves: Canal Messier, diamicton masivo, explanada de represamiento, manto, banco morrénico, outwash submarino.

\section{Sequence of submarine depositional landforms in the Messier Channel fracture, Central Patagonia}

\begin{abstract}
The sequence and location of the bottom depositional landforms are analysed, in order to find phases of the morphogenesis. The distribution of landforms was established through a longitudinal profile by means of high resolution echo sounder records and in accordance with their decreasing oldness.

Along Messier Channel, two types of submarine zone appear: a) Where confluent fjords are absent, bottoms are shallow (-50 to $-450 \mathrm{~m}$ ); landforms of massive diamicton are interstratified with laminated beds of ponding esplanades in the depressions, meanwhile thin ponded structures predominate in the shallow basins; the fundamental stratigraphic reference is a drape of sediments, which overlies all the bottom features. b) Where confluent fjords are present, the excavation landforms are the deepest $(-50$ to $-1350 \mathrm{~m})$; the landforms of massive diamicton interstratified with the beds of the bottom ponding esplanades are best developed; as stratigraphic reference, the regional drape of cover lacks in these deepest troughs.

Consequently, the covering drape seems to have been installed when the ice remained yet facing each fjord, but when it had abandoned the zone of shallow bottoms. These features are thought to be related with a rapid glacial retreat during Late Glacial times evidenced by former works.
\end{abstract}

Key words: Messier Channel, massive diamicton, ponding esplanade, drape, morainal bank, submarine outwash. 


\section{INTRODUCCIÓN}

La operación terrestre de la deriva glacial en la Ultima Glaciación fue documentada para el piedmont de Chiloé de Norpatagonia (Heusser y Flint, 1977; Heusser, 1990), de lo que se deduce que si el hielo cubrió el paisaje hacia el W hasta la isla de Chiloé, debió hacerlo por lo menos en un área semejante hacia el sur. Después de dos máximos, datados en 20. 000 a 19.000 años A.P. y en 14.890 a 13.900 años A.P. $C^{14}$ (Lowell et al., en Heusser, 1997), la deglaciación debe haber sido rápida, porque el clima se tornó abruptamente más cálido (Heusser, 1990), llegando algunos glaciares a ser más pequeños que en el presente hacia los 11.000 años A.P. $\mathrm{C}^{14}$ (Mercer, 1970). En torno a esta última edad hubo también impactos de volcanismo explosivo (Lumley, 1993, en Heusser, 1997).

Se puede asumir que el efecto de la Ultima Glaciación en la Patagonia Central debe haber sido un poco más fuerte que en Norpatagonia. Aún así, tres cores del R/V Polar Duke obtenidos entre 330 y $730 \mathrm{~m}$, dan edades $\mathrm{C}^{14}$ no corregidas de 3.000 a 9.000 años para espesores de sedimentos de 5 a $8 \mathrm{~m}$ de subfondo (Leventer et al., 1995), cerca de los extremos del Canal Messier y hacia el frente oceánico archipielágico; ello indica edad holocénica para subfondos comparables.

Hasta ahora, sólo se conocía rasgos de la expresión morfogenética submarina de estos fenómenos para la depresión submarina y fractura del Canal de Moraleda en Norpatagonia (Araya-Vergara, 1997, 1998), donde se vio que sus rasgos tienen relación directa con la ausencia o presencia de fiordos confluentes.

En consecuencia, el propósito de este trabajo es extender este tipo de conocimientos hacia el sur, establecer la secuencia espacio-temporal de las formas deposicionales del fondo de la fractura longitudinal de Patagonia Central, y tratar de encontrar indicaciones para el estudio de las fases morfogenéticas glacio-marinas que las expliquen.

\section{MATERIALES Y MÉTODOS}

El perfil longitudinal de Canal Messier (Fig. 1) se obtuvo de registros de un ecosonda Edo Western, modelo 248E, que operó en el modo de perfilador de subfondo (frecuencia $3,5 \mathrm{kHz}$ ), a bordo del AGOR Vidal Gormaz, durante el crucero de Campos de Hielo Sur. La velocidad del buque no fue anotada regularmente sobre el registro, de modo que su escala horizontal fue calculada con ayuda del perfil logrado por digitalización a nivel topográfico (CONA, 1995), resultando un valor medio de 1 : 60.000 , que da un factor de 20 para la exageración de la escala vertical, de 1: 3.000. Para compensar los posibles efectos en longitud de pulso en el registro, por cambios de velocidad no conocidos, se correlacionó los reflectores según el criterio expuesto en Araya-Vergara (1997), otorgando más importancia a las estructuras de conjunto de reflectores que a aquellos individuales. Para resaltar detalles en estas estructuras, las fotos del registro fueron digitalizadas por scanner y procesadas computacionalmente con los programas Microsoft Photo Editor y Corel Photo Paint, aplicando ajustes de contraste, brillo, corrección Gamma y selección de escenas ampliadas.

Para facilitar la interpretación, se agregó a las imágenes una escala gráfica de pendientes, según la técnica usada en Araya-Vergara (1997) que incluye valores en altan (Young, 1975) para normalizar su comparación.

El reconocimiento de la naturaleza aproximada de los materiales, en función de la forma del fondo, se basó someramente en la información disponible de muestras de sedimentos obtenidos con box corer, que corresponden a limos (Ríos y Cisternas, 1998), por representar sólo la naturaleza del fondo. Por lo tanto, la información de subfondo se extrajo esencialmente con métodos acústicos.

Para interpretar las superficies reflectoras en términos de la naturaleza de los materiales, se consideró que el sonar de $3,5 \mathrm{kHz}$ posee gran variabilidad de penetración de decenas de metros en sustratos fangosos blandos, a unos pocos metros en sustratos arenosos (Carlson, 1989). La estructura de ecos fue interpretada de acuerdo con la experiencia y teoría de observación de capas laminadas (p. ej. Carlson, 1989), de ecos incoherentes en formas caóticas en relación con capas laminadas (Josenhans y Fader, 1989), de sistemas complejos con derivación de modelos (Shaw, 1971; Powell, 1984, en Guilcher, 1985; Mc Cabe, 1986; Barret y Hambrey, 1992; Henderson, 1988; Kaszycki, 1987; Brodzikowski, 1993; Auton, 1992) y de mantos (drapes) submarinos (Chiocci et al., 1990; Hine et al., 1994; Alvarez et al., 1985).

En sectores de umbrales rocosos y detríticos, se consideró la probable influencia de ecos falsos o de rasgos de los flancos del transecto del buque, alcan- 


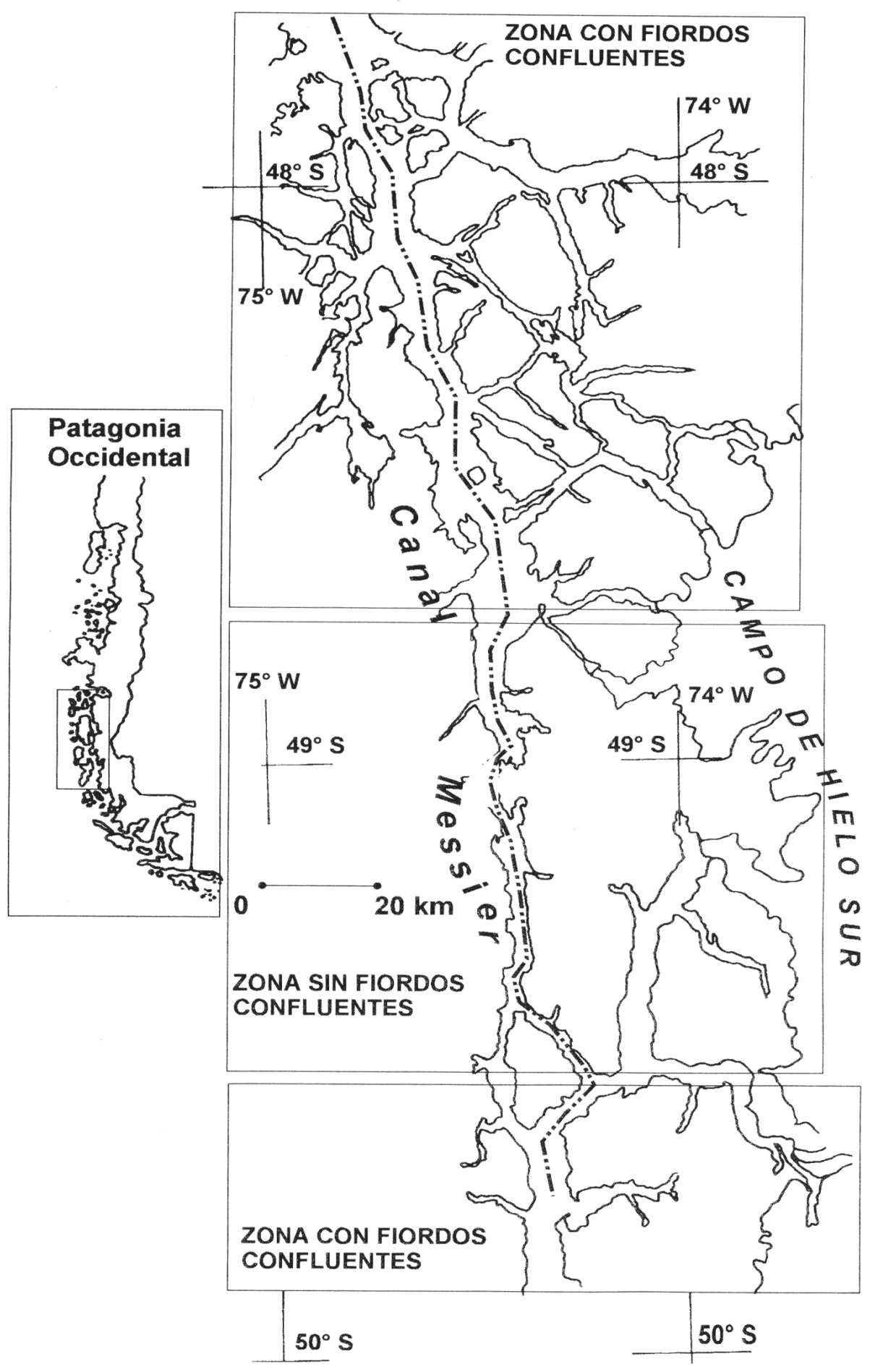

Figura 1. Localización geográfica del área de estudio: perfil longitudinal y zonas. Figure 1. Geographic location of study area: long profile and zones. 
zados por el haz acústico, que podía alterar la interpretación, para esto se trabajó con la clasificación de caracteres acústicos de Damuth (1978), aplicada a registros con pulsos a $3,5 \mathrm{kHz}$ y basada fundamentalmente en la "prolongación" de la señal de retorno y la observación de rasgos hiperbólicos, su interpretación genética fue posible gracias a experiencias recopiladas por Embley y Langseth (1977), Damuth (1978), Karl (1989), Pratson y Laine (1989), Uchupi et al. (1996), Araya-Vergara (1998), y otros; ellas indican que los efectos acústicos por complicaciones técnicas pueden ponderarse con un seguimiento regional de las estructuras acústicas; esto es especialmente aplicable cuando hay similar "prolongación" entre diamicton masivo y basamento en umbrales; la masa de diamicton presenta estructura microhiperbolada a blocosa, indicando sus deformaciones, contrastando con el eco prolongado nítido de las macrohipérbolas que representan el basamento acústico.

\section{RESULTADOS}

A lo largo de Canal Messier, la superficie reflectora que representa el basamento acústico muestra dos zonas diferenciadas por la ausencia o presencia de fiordos confluentes desde el este (Figs. 1 a 3). La zona central (Fig. 2), carente de fiordos confluentes, coincide con la parte más estrecha del canal; su excavación es poco profunda, pero con disección densa (Figs. 4 y 5). La zona de los sectores sur y norte (extremo sur en Figs. 2 y 3), con fiordos confluentes, presenta excavación profunda y corresponde a la parte más ancha de la fractura del canal; especialmente el sector norte muestra bien la excavación en valles de fiordos confluentes hasta profundidades superiores a $1000 \mathrm{~m}$ (Figs. 6 a 9).

Por consiguiente, la descripción de las morfoestructuras se hará por separado para cada zona y por orden de su antigüedad decreciente. $\mathrm{La}$ Tabla 1 esquematiza el contenido de las Figs. 1 a 9.

\section{Zona sin fiordos confluentes} (Tabla 1; Figs. 2, 4 y 5).

\section{Diamicton masivo (Figs. 3 y 5)}

La concordancia de nivel de cimas y de depresión poco profundas, está representado por reflectores incoherentes y sólo existe en las mayores depresiones. Su mayor antigüedad se deduce de dos hechos: ocupa tanto las vertientes como el fondo de depre- siones, sobreyaciendo directamente a la superficie del basamento acústico, en forma de diamicton de umbral o de explanada; está interestratificado con las capas laminadas de la explanada de represamiento en forma de diamicton tongue (lengua de diamicton), de lo que se deduce que éstas provienen de él.

\section{Explanada de represamiento cautivo (Fig. 5)}

La potencia total del conjunto de capas laminadas varía entre 6 y $35 \mathrm{~m}$. El número de capas reconocibles varía entre 2 y $5 \mathrm{~m}$ y su espesor medio entre 3,5 y $8 \mathrm{~m}$. La estratificación tiende a ser gradual, pasando de una parte inferior acústicamente transparente a una más delgada representada por un eco nítido en cada capa. Esto es consistente con la relación de interestratificación con el diamicton masivo. Comúnmente sobreyace a un diamicton masivo basal, cuya potencia visible varía entre 7 y $35 \mathrm{~m}$. Por lo tanto, secuencialmente, se trata a la vez de postdiamicton y de forma sincrónica a él. En todo caso, es la forma temporalmente siguiente a las generadas directamente por diamicton masivo; se denomina cautiva, por estar totalmente recubierta por el manto (drape) de fondo.

\section{Drape (manto) de fondo (Figs. 4 a 6)}

Se trata de una capa acústicamente transparente, de 4 a $17 \mathrm{~m}$ de espesor, que cubre como manto a las formas deposicionales de fondo en las diferentes cubetas. Es una importante capa guía, porque sella la fase de la explanada de represamiento, transformándola en forma cautiva en el fondo estrecho de las cubetas. En su superficie, un eco delgado (1 m?) indica una fase tardía de depositación de sedimentos finos limosos (ver materiales y métodos).

\section{Drape (manto) de vertiente y fenómenos de remoción en masa (Figs. 4 a 6 )}

A juzgar por la frecuente forma cóncava del drape (manto) de fondo y su engranamiento con el de vertiente, mediante una concavidad basal, se puede deducir que ambos mantos forman uno solo en este paisaje submarino. En éste, los fenómenos de remoción en masa deben ser deducidos teniendo en cuenta las pendientes de funcionamiento (ver escalas de pendientes en las figuras). En pendientes entre 16 y $32^{\circ}$, en un rango entre 2,5 y 3 altanes, se encuentran accidentes en las superficies reflectoras que representan el manto: separación en bloques rectangulares, deformación de la estructura y deformación máxima en la base. Estas estructuras han sido inter- 
FRACTURA LONGITUDINAL DE CANAL MESSIER

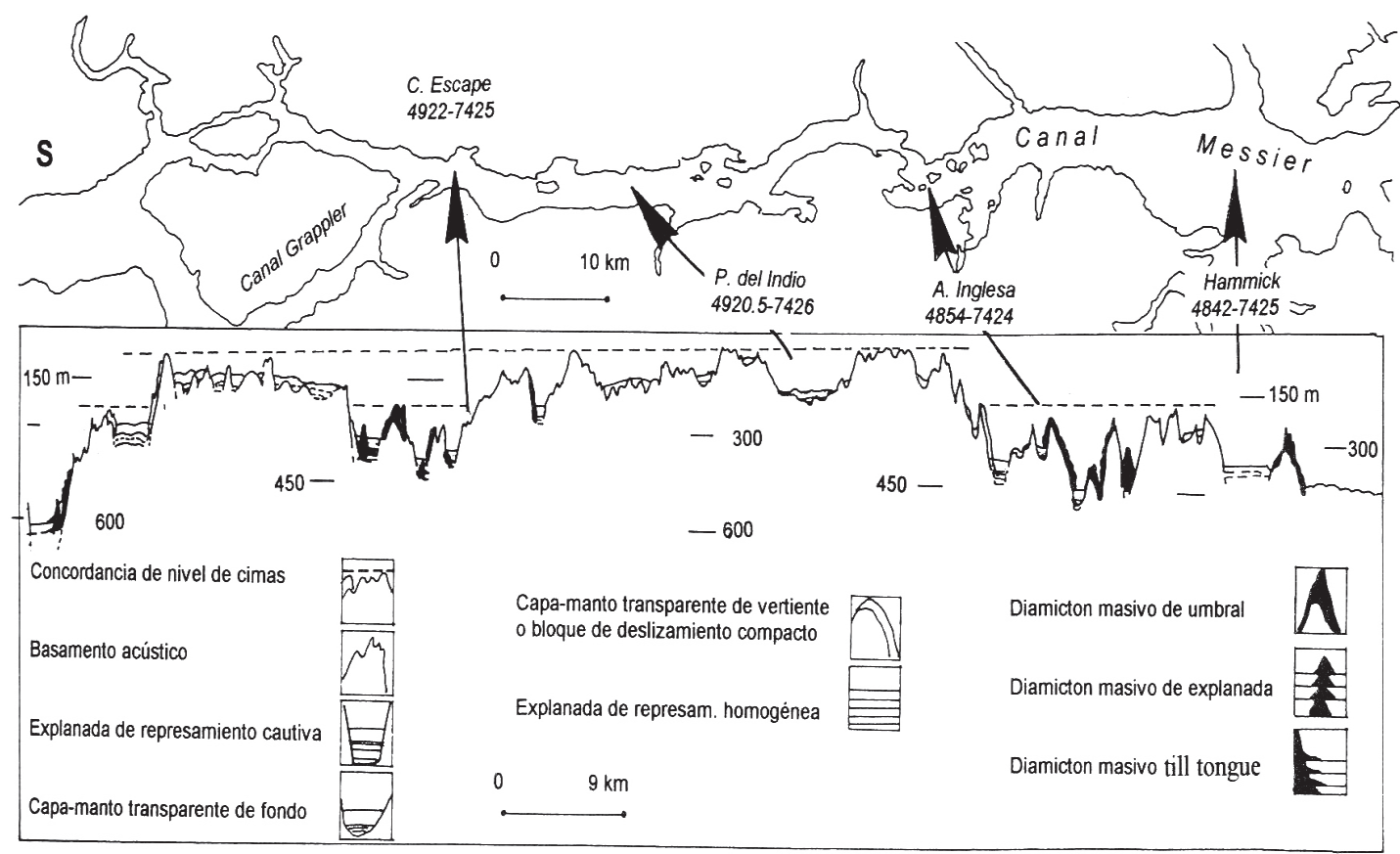

Figura 2. Zona sin fiordos confluentes.

Figure 2. Zone without confluent fjords.

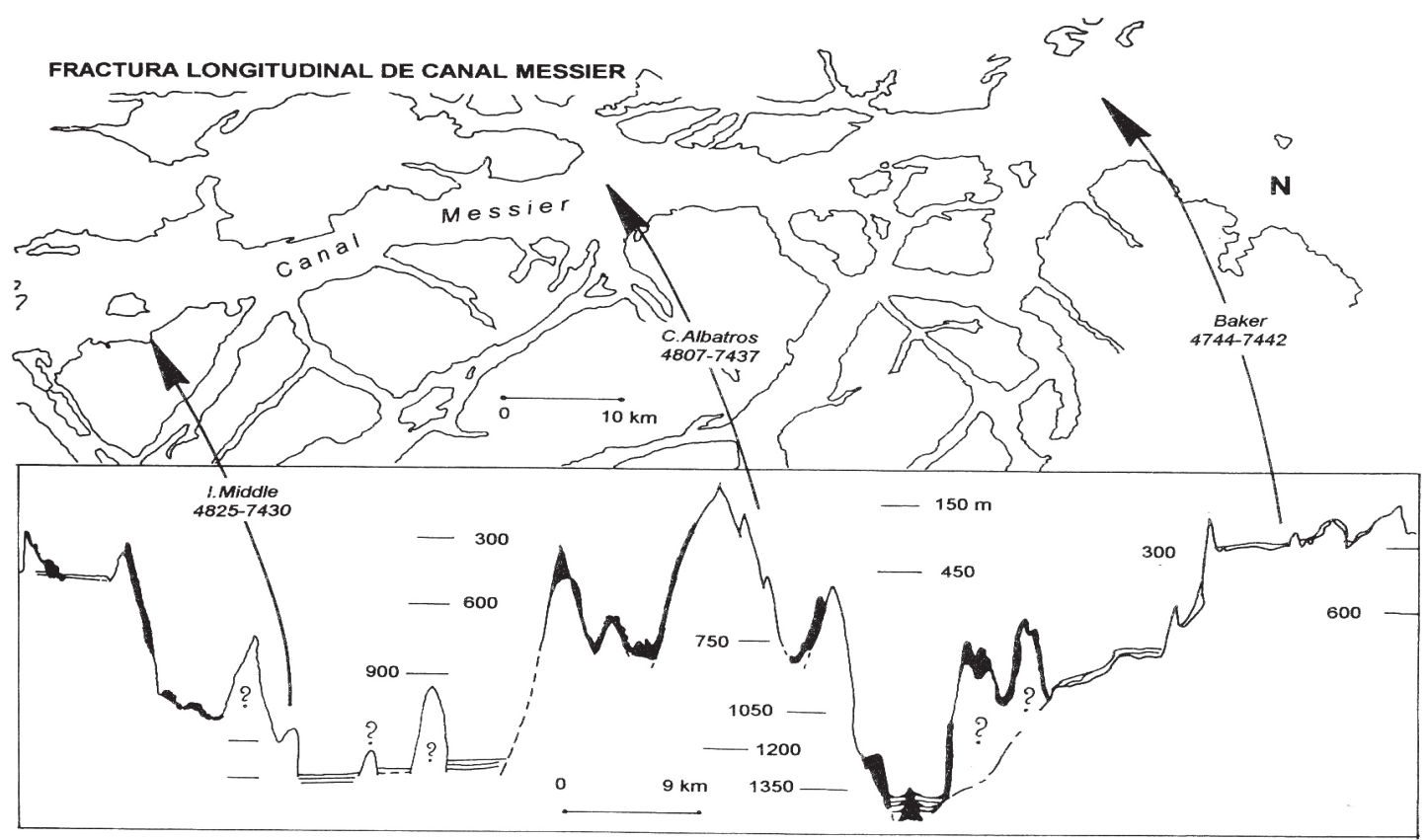

Figura 3. Zona con fiordos confluentes. Usar leyenda de Fig. 2.

Figure 3. Zone with confluent fjords. Use legend of Fig. 2. 
Tabla 1: Zonación y secuencia de morfoestructuras; la diferencia secuencial está indicada por el estilo de la letra: formas antiguas, formas medias y formas recientes.

Table 1: Zonation and sequence of morphostructures; sequential difference is indicated by the typographic style: ancient, average and recent forms.

\begin{tabular}{|c|c|c|c|c|}
\hline ZONA & $\begin{array}{l}\text { Profundidad de } \\
\text { concordancia de } \\
\text { nivel de cimas } \\
\text { de umbral }\end{array}$ & $\begin{array}{l}\text { Profundidad de } \\
\text { fondo de depresión }\end{array}$ & $\begin{array}{l}\text { Formas de umbral y } \\
\text { vertiente }\end{array}$ & $\begin{array}{l}\text { Formas de fondo } \\
\text { y de base de } \\
\text { vertiente }\end{array}$ \\
\hline \multirow[t]{2}{*}{$\begin{array}{l}\text { SIN FIORDOS } \\
\text { CONFLUENTES } \\
\text { (estrecha y } \\
\text { poco profunda) }\end{array}$} & $\begin{array}{l}\text { Aprox. } 50 \mathrm{~m} \\
\text { (nivel somero) }\end{array}$ & $\begin{array}{l}\text { Aprox. } 150 \mathrm{~m} \\
\text { (depresiones } \\
\text { someras) }\end{array}$ & $\begin{array}{l}\text { - } \frac{\text { Bloque de }}{\text { deslizamiento }} \\
\text { - } \underline{\text { compacto }} \\
\underline{\text { Manto dudoso }}\end{array}$ & $\begin{array}{l}\text { - } \frac{\text { Manto superficial - }}{\text { concavidad basal }} \\
\text { - } \frac{\text { Manto de fondo- }}{\text { abombamiento }} \\
\text { - } \begin{array}{l}\text { Explanimal } \\
\text { represamiento de } \\
\text { probable }\end{array}\end{array}$ \\
\hline & $\begin{array}{l}\text { Aprox. } 200 \mathrm{~m} \\
\text { (nivel poco } \\
\text { profundo) }\end{array}$ & $\begin{array}{l}\text { Aprox. } 450 \mathrm{~m} \\
\text { (depresiones } \\
\text { poco profundas) }\end{array}$ & $\begin{array}{l}\text { - } \frac{\text { Deslizamiento con }}{\text { sedimentos }} \\
\text { remoldeados } \\
\text { Bloque de } \\
\frac{\text { deslizamiento }}{\text { compacto }} \\
\text { - } \text { Manto } \\
\text { Diamicton masivo }\end{array}$ & $\begin{array}{l}\text { - } \frac{\text { Manto superficial - }}{\text { concavidad basal }} \\
\text { - } \frac{\text { Manto-punta }}{\text { de compresión }} \\
\frac{\text { de deslizamiento- }}{\text { abombamiento }} \\
\text { proximal } \\
\text { Explanada de } \\
\text { represamiento } \\
\text { cautiva } \\
\text { - Diamicton masivo } \\
\text { de explanada- } \\
\text { lengua de } \\
\text { diamicton }\end{array}$ \\
\hline $\begin{array}{l}\text { CON FIORDOS } \\
\text { CONFLUENTES } \\
\text { (ancha y profunda) }\end{array}$ & $\begin{array}{l}\text { Aprox. } 300 \mathrm{~m} \\
\text { (nivel profundo) }\end{array}$ & $\begin{array}{l}\text { Aprox. } 1000 \text { a } 1350 \mathrm{~m} \\
\text { (depresiones } \\
\text { profundas) }\end{array}$ & - Diamicton masivo & $\begin{array}{l}\text { - Explanada de } \\
\text { represamiento } \\
\text { - Diamicton masivo } \\
\text { de explanada- } \\
\text { lengua de } \\
\text { diamicton }\end{array}$ \\
\hline
\end{tabular}

pretadas fundamentadamente como bloques de deslizamiento compacto, sedimentos remoldeados por deslizamiento y puntas de compresión de deslizamiento (ver autores citados en Materiales y Métodos). Estos rasgos son post-sedimentarios (epigénicos) y posteriores -al menos- al manto de fondo, puesto que éste está deformado convexamente por empuje en la forma que ha sido denominada abombamiento proximal, por ubicarse próxima a la vertiente (un caso particularmente notable se describe en la Fig. 6).

\section{Zona con fiordos confluentes (Tabla 1; Figs. 5} a 8)

\section{Diamicton masivo}

Los ecos incoherentes que lo representan se encuentran tanto en umbrales como en los fondos de cubetas profundas. Su contacto directo con la superficie rocosa del basamento convierte a las formas que lo contienen en las más antiguas. Sus materiales están interestratificados en (lengua de diamicton) con las capas laminadas de la explanada de represamiento.

\section{Explanada de represamiento}

$\mathrm{Su}$ forma es la más parecida a sus homólogas de fiordos confluentes. La potencia del conjunto de 


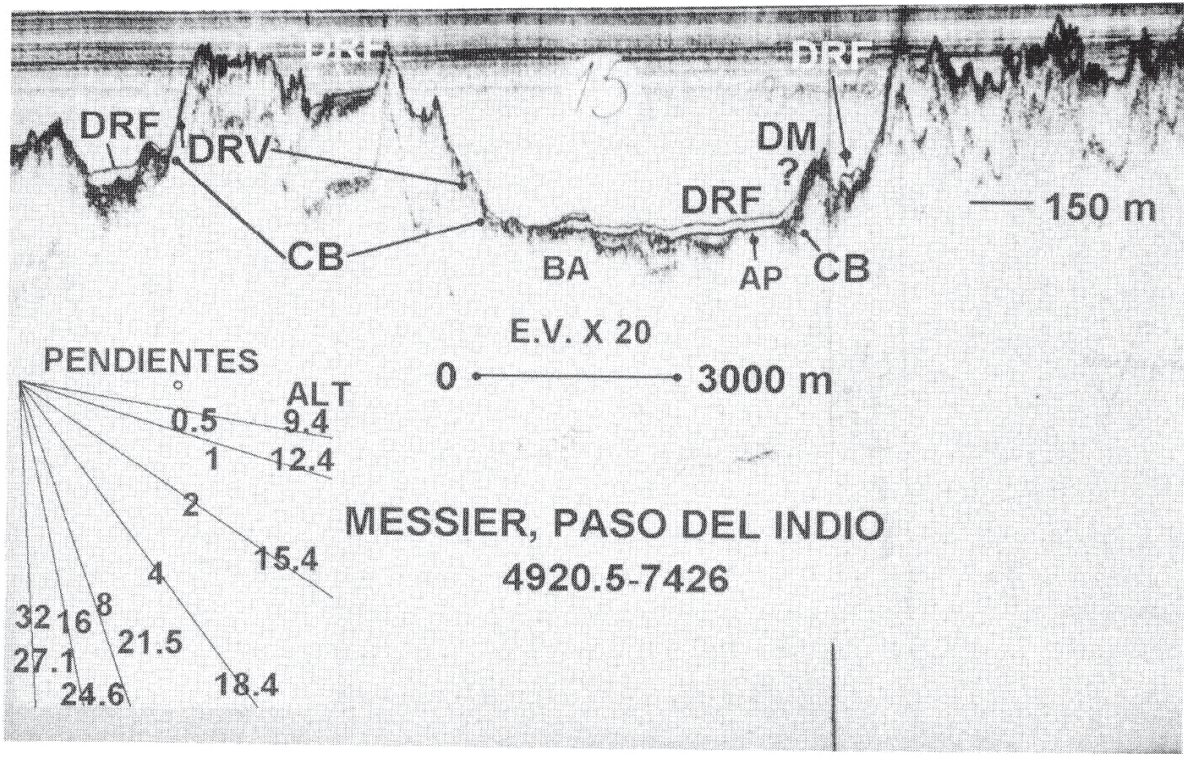

Figura 4. Zona sin fiordos confluentes. DM? = diamicton masivo dudoso; $\mathbf{B A}$ = basamento acústico (formas erosionales rocosas); $\mathrm{DRF}=$ drape (manto) de fondo; $\mathrm{CB}=$ concavidad basal; $\mathbf{A P}=$ abombamiento proximal; DRV = manto de vertiente.

Figure 4. Zone without confluent fjords. DM? = doubtful massive diamicton; BA = acoustic basement (rocky erosional landforms); $\mathrm{DRF}=$ bottom drape; $\mathrm{CB}=$ basal concavity; $\mathrm{AP}=$ proximal bending; $\mathrm{DRV}=$ slope drape.

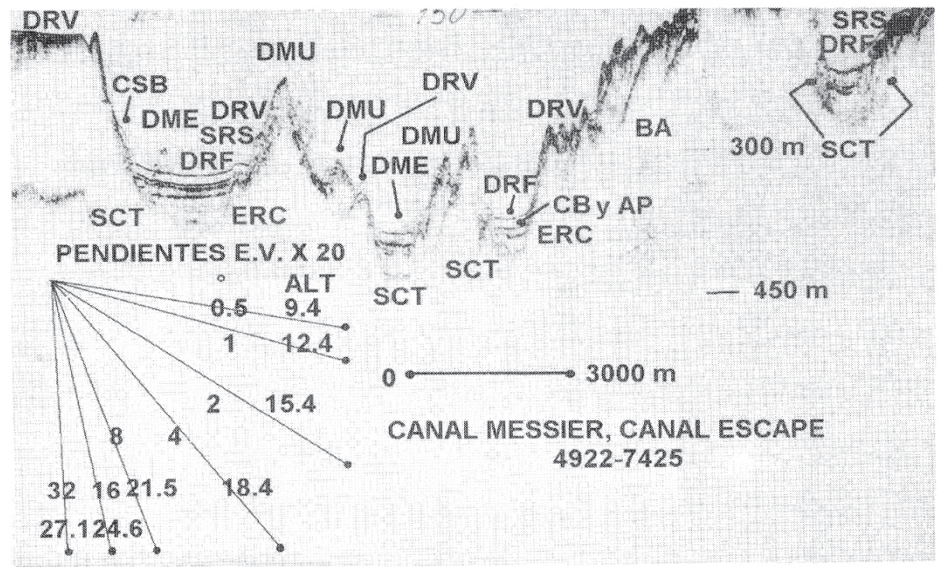

Figura 5. Zona sin fiordos confluentes. $\mathrm{BA}=$ basamento acústico; $\mathrm{DMU}=$ diamicton masivo de umbral; DME = diamicton masivo de explanada; $\mathrm{ERC}=$ explanada de represamiento cautiva; $\mathrm{DRF}=$ manto de fondo;

DRV = manto de vertiente $\mathbf{C B}=$ concavidad basal $; \mathrm{CSB}=$ deslizamiento en bloque compacto; $\mathbf{S R S}=$ deslizamiento con sedimentos remoldeados; $\mathrm{AP}=$ abombamiento proximal; $\mathrm{SCT}=$ punta de compresión de deslizamiento.

Figure 5. Zone without confluent fjords. $\mathrm{BA}=$ acoustic basement; $\mathrm{DMU}=$ threshold massive diamicton; $\mathrm{DME}=$ esplanade massive diamicton; $\mathrm{ERC}=$ captive ponding esplanade; $\mathrm{DRF}=$ bottom drape; $\mathrm{DRV}=$ slope drape; $\mathrm{CB}=$ basal concavity; $\mathrm{CSB}=$ compact slide blocks; $\mathrm{SRS}$ = slide remolded sediments; $\mathrm{AP}$ = proximal bending; $\mathrm{SCT}=$ slide compression toe. 


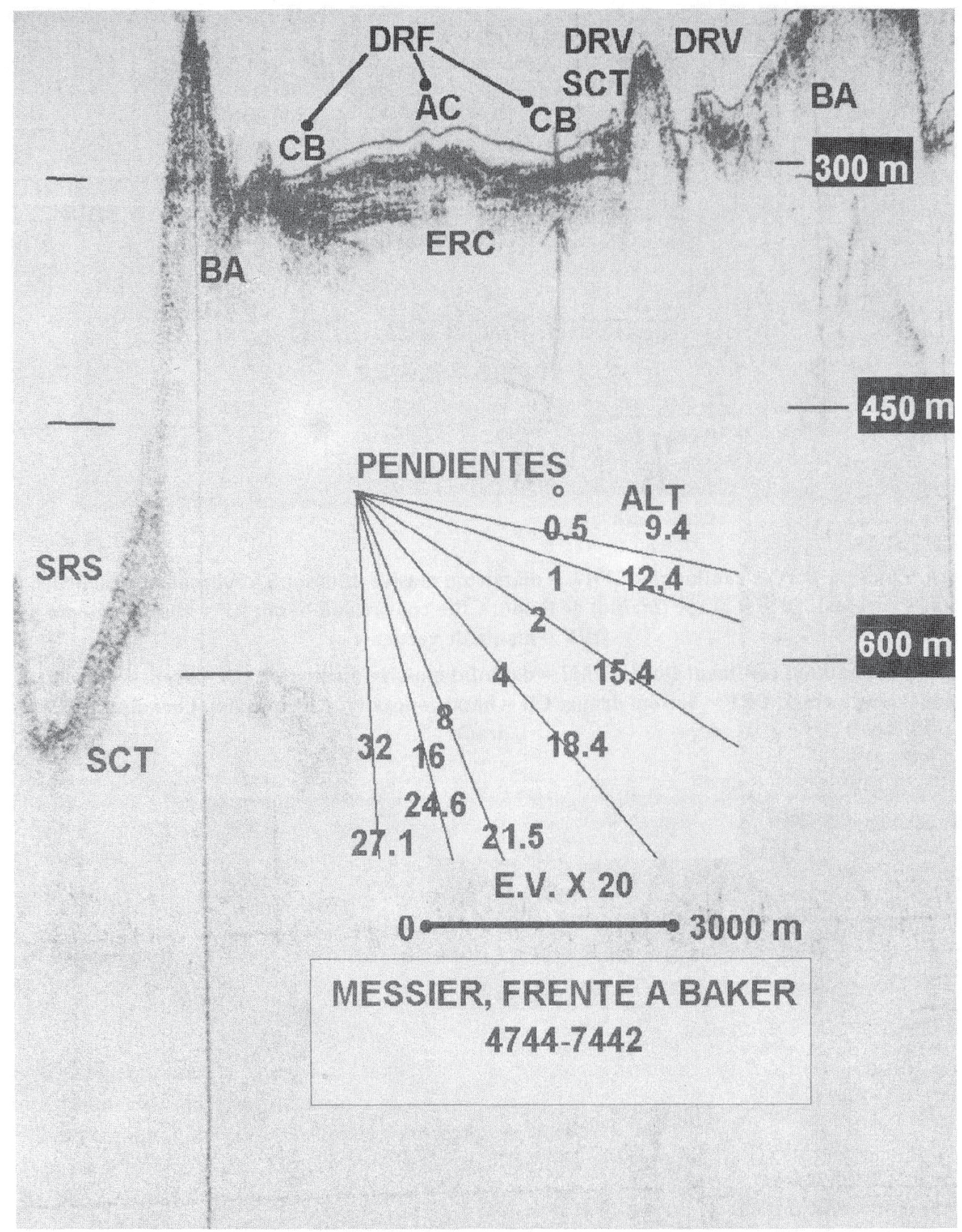

Figura 6. Zona con fiordos confluentes. DRF = manto de fondo; CSB = deslizamientos en bloque compacto; BA = basamento acústico; $\mathrm{SRS}$ = deslizamiento con sedimentos remoldeados; $\mathrm{SCT}=$ punta de compresión de deslizamiento; $\mathrm{AP}=$ abombamiento proximal; $\mathrm{DMU}=$ diamicton masivo de umbral; $\mathrm{DME}=$ diamicton masivo de explanada; DMTT = lengua de diamicton.

Figure 6. Zone with confluent fjords. DRF = bottom drape; $\mathrm{CSB}=$ compact slide block; $\mathrm{BA}=$ acoustic basement; $\mathrm{SRS}=$ slide remolded sediment SCT = slide compression toe; $\mathrm{AP}=$ proximal bending; $\mathrm{DMU}=$ threshold massive dimicton; DME = esplanade massive diamicton; DMTT = diamicton tongue. 


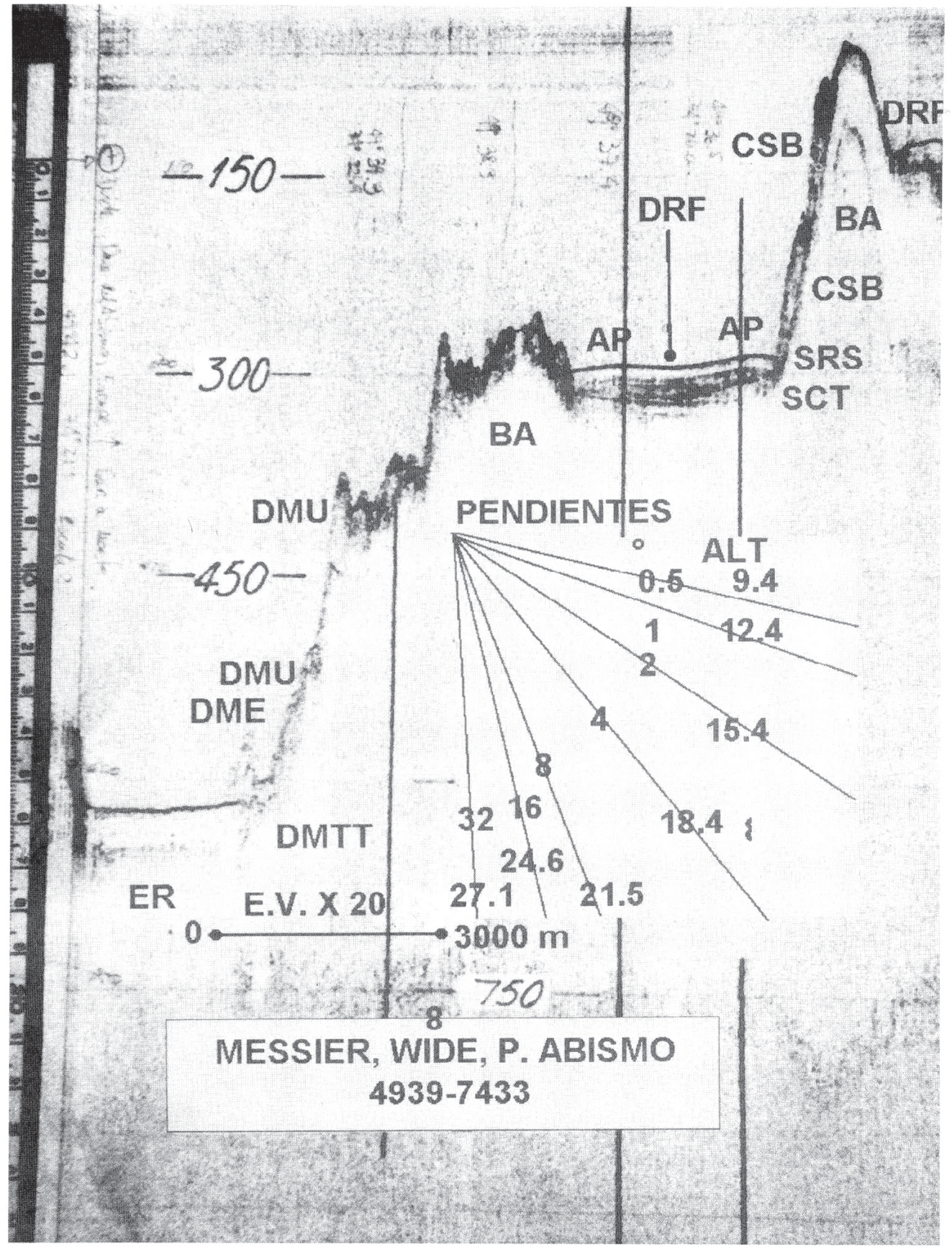

Figura 7. Zona con fiordos confluentes. $\mathrm{BA}=$ basamento acústico; $\mathrm{ERC}=$ explanada de represamiento cautiva; DRF = manto de fondo; DRV = manto de vertiente; $\mathrm{SCT}$ = punta de compresión de deslizamiento; $\mathrm{CB}=$ concavidad basal; $\mathrm{AC}=$ abombamiento central.

Figure 7. Zone with confluent fjords. $\mathrm{BA}=$ acoustic basement; $\mathrm{ERC}=$ captive ponding esplanade; $\mathrm{DRF}=$ bottom drape; $\mathrm{DRV}=$ slope drape $\mathbf{S C T}=$ slide compression toe $; \mathrm{CB}=$ basal concavity $\mathbf{A C}=$ central bending . 


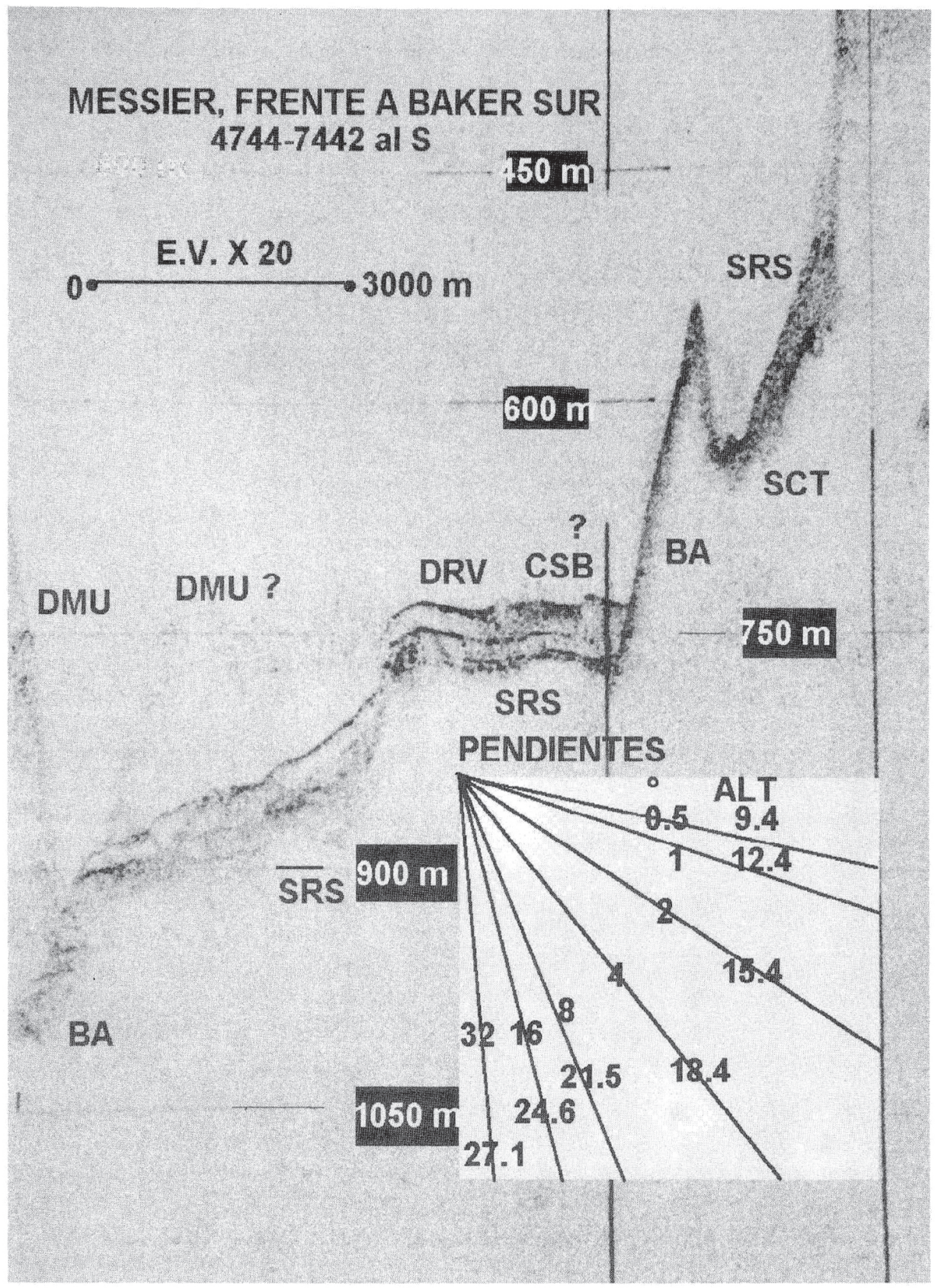

Figura 8. Zona con fiordos confluentes. $B A=$ basamento acústico; DRV= manto de vertiente; CSB?= probables bloques de deslizamiento compacto; $\mathrm{SRS}=$ deslizamiento con sedimentos remoldeados; $\mathrm{SCT}=$ punta de compresión de deslizamiento; DMU?= diamicton masivo de umbral probable; $D M U=$ umbral alto de diamicton masivo.

Figure 8. Zone with confluent fjords. $B A=$ acoustic basement; $D R V=$ slope drape; CSB?= probable compact slide block; $\mathrm{SRS}=$ slide remolded sediments; $\mathrm{SCT}=$ slide compression toe; $\mathrm{DMU} ?=$ probable threshold massive diamicton; $\mathrm{DMU}=$ high threshold massive diamicton. 


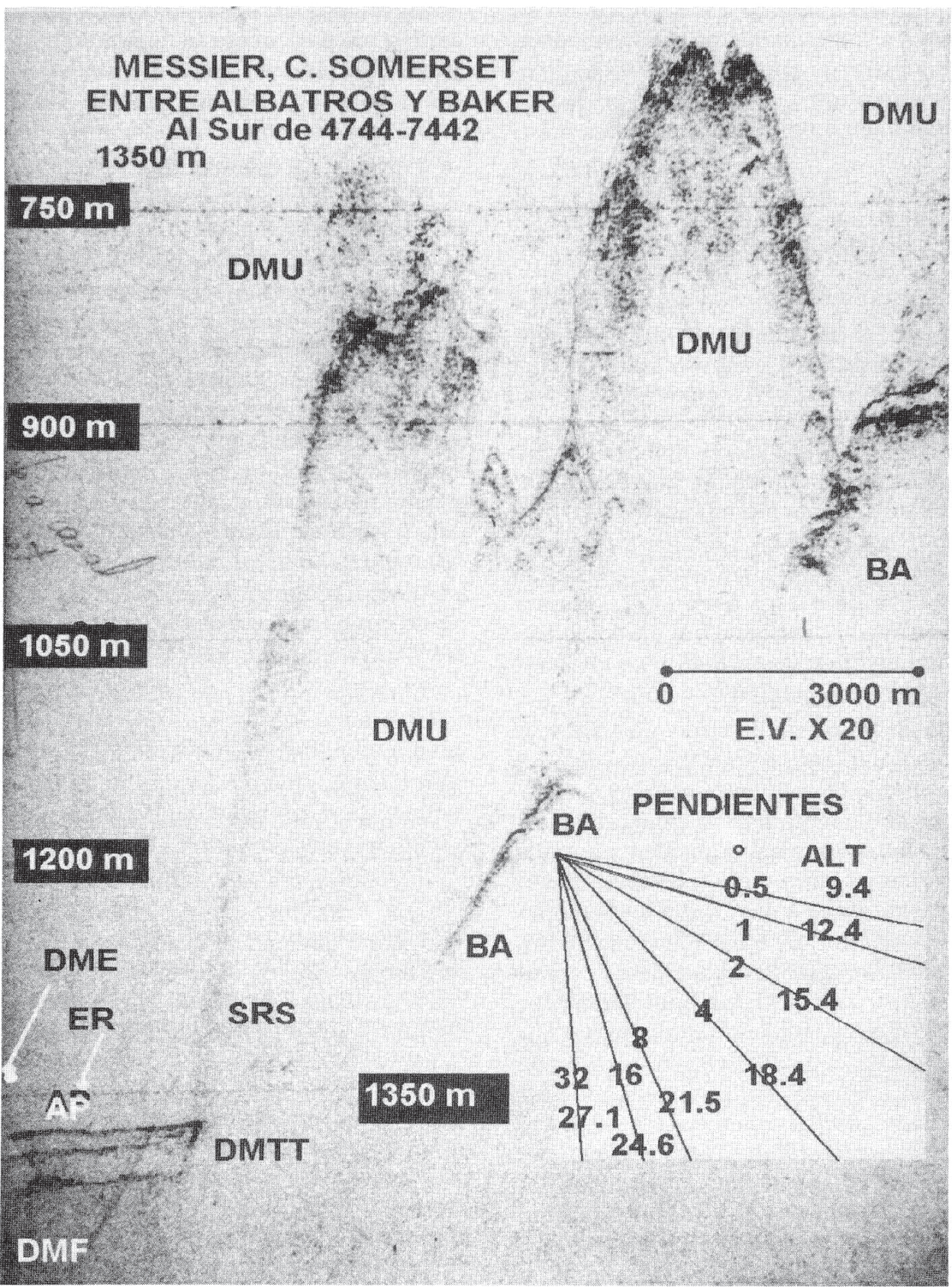

Figura 9. Zona con fiordos confluentes. BA= basamento acústico; DMU= diamicton masivo de umbral; $\mathrm{SRS}=$ sedimentos remoldeados por deslizamiento; $\mathrm{DMF}$ = diamicton masivo de fondo; $\mathrm{DMTT}=$ lengua de diamicton; $\mathbf{E R}=$ explanada de represamiento; $\mathrm{DME}=$ diamicton masivo de explanada; $\mathrm{AP}=\mathbf{a b o m b a m i e n t o}$ proximal.

Figure 9. Zone with confluent fjords. $B A=$ acoustic basement; $D M U=$ threshold massive diamicton; $S R S=$ slide remolded sediments; $\mathrm{DMF}=$ bottom massive diamicton; $\mathrm{DMTT}=$ diamicton tongue; $\mathrm{ER}=$ ponding esplanade; $\mathrm{DME}=$ esplanade massive diamicton; $\mathbf{A P}=$ proximal bending. 
capas laminadas es desconocida; su interestratificación con el diamicton masivo mediante lengua de diamicton se aprecia observando directamente el registro original; por lo tanto, se deduce que es inmediatamente posterior al material masivo.

\section{DISCUSIÓN}

Las formas descritas asociadas al diamicton masivo y su relación con las explanadas de represamiento han sido interpretadas como glaciales y glaciomarinas (Carlson, 1989; Josenhans y Fader, 1989; Araya-Vergara 1997; 1998). Ello es consistente con las observaciones y derivaciones de modelos sobre interacciones complejas de la deriva glacial en facies marinas y lacustres (Shaw 1971; Mc Cabe 1986; Barrett y Hambrey 1992; Brodzikowski 1993). Considerando los resultados obtenidos en la costa (Mercer 1970; Heusser y Flint 1977; Heusser 1990, 1997), es posible establecer el grado de importancia de la morfogénesis glacial y su localización relativa en el área estudiada.

Las formas atribuibles a la deriva glacial están representadas sólo en las profundas excavaciones de la zona con fiordos confluentes y en las cubetas más deprimidas de la zona sin fiordos confluentes; las colinas de diamicton masivo pueden ser interpretadas como bancos morrénicos (morainal banks) y las explanadas de represamiento que los acompañan, como resultado de outwash submarino (submarine outwash), según el esquema de Powell y Molnia (1989) y los modelos publicados por Anderson (1993); las lenguas de diamicton que conectan ambas formas coinciden con el esquema de lengua de till (till tongue) de Josenhans y Fader (1989). Las edades de los espesores de subfondo obtenidas de los cores del Polar Duke (Leventer et al., 1995), indican que el outwash submarino debe haberse instalado tanto en el Pleistoceno Reciente como en el Holoceno, porque la base de éste está sólo a unos 10 o $20 \mathrm{~m}$ del fondo, sobreyaciendo a capas laminadas que conservan el mismo estilo estratigráfico, lo que revela que el abastecimiento de materiales desde los bancos morrénicos ha sido continuo en ambas épocas. Tanto cubetas como umbrales de aguas someras carecen de este sistema de formas. En ellas, en cambio, dominan las formas recientes de manto (drape) y sus consecuentes derivadas de la remoción en masa, todas más recientes que las de deriva glacial. La falta de rasgos glacigénicos submarinos en este orden de profundidad, puede pensarse relacionada con la mayor antigüedad del retroceso glacial, y con el tiempo y condiciones necesarios para que sus depósitos fueran redistribuidos como manto en medio marino.

Por lo tanto, la fractura del Canal Messier parece mostrar dos grandes fases morfogenéticas; una correspondiente a la deriva glacial y otra, a la formación de un manto de sedimentos cubriendo al paisaje, acompañada o seguida por movimientos en masa. La primera, más antigua, debe haber tenido manifestaciones tardías en las áreas más profundas, no sólo porque allí están los rasgos característicos de deriva glacial, sino también por la carencia de manto. Esto puede ser interpretado del siguiente modo: aun cuando la fase de construcción del manto es posterior a la glacigénica, aquélla debe haberse estabilizado cuando el hielo, después de retirarse de las partes someras, permaneció sólo en los valles profundos; sobre todo frente a los actuales fiordos, cuyos rasgos de fondo indican retroceso glacial tardío (Araya-Vergara, 1997).

El desarrollo libre de las formas glacigénicas es consistente con la gran profundidad de su emplazamiento, donde el glaciar debe haber operado como tabla de hielo flotante (ice shelf); este modo es contemplado en los modelos de Anderson y Molnia (1989, en Anderson, 1993) y de Barret y Hambrey (1992), acerca de zonación morfogenética bajo ice shelf, en función de la distancia al borde de la línea de varadura (grounding line). En estos modelos se asume que la zona de diamicton masivo es la más cercana a este borde, apareciendo más distante el diamicton estratificado; representaciones de Powell y Molnia (1989) indican la formación de bancos morrénicos en el término del glaciar sobre la línea de varadura, apareciendo el diamicton estratificado como outwash marino, distal al banco morrénico; conjuntamente, la liberación de till balseado por icebergs puede haber operado como una fuente importante de diamicton masivo, si se aplican los modelos y representaciones de Anderson (1993) y Domack (1990) y las observaciones específicas de Dowdeswell et al. (1994).

Es muy probable que la mayor permanencia del hielo frente a los fiordos confluentes haya impedido la formación del manto. La presencia de éste en fondos someros puede estar relacionada con la rápida deglaciación y oscilaciones deducidas de los resultados de Heusser (1997) y Mercer (1970); para la comprensión de los procesos que hicieron posi- 
ble la diseminación del material del manto puede acudirse a modelos que integran plumas turbias manifestadas, como overflow, interflow (Anderson y Molnia, 1989, en Anderson 1993; Stewart, 1988, en Lemmen, 1990; Domack, 1990) y aún como underflow relacionado con corrientes de turbidez, producidos en frecuentes ambientes de debacle.

La profundidad relativa del fondo cuando el hielo operaba no parece haber sido muy diferente a la actual, balanceando la regresión glacioeustática y el hundimiento glacioisostático. Mediciones de solevantamiento en Chiloé continental para algo más de 3.000 años, dan una tasa máxima de $8,2 \mathrm{mka}^{-1}$ que, proyectada especulativamente en el Holoceno como tiempo de relajación isostático, da $82 \mathrm{~m}$ de solevantamiento probable (Hervé y Ota, 1993). Esto sugiere que el espesor del hielo en la Última Glaciación puede relacionarse con profundidades poco diferentes a las actuales, lo que tiende a validar la interpretación de la morfogénesis con hielo en ice shelf, aún extrapolando los espesores glaciales estimados por Porter (1981, en Heusser, 1990).

\section{CONCLUSIONES}

Es probable que estas observaciones puedan ser usadas para el estudio de estadios del Pleistoceno y de la influencia del Holoceno en la morfogénesis de estos ambientes submarinos. Los rasgos de la zona con fiordos confluentes parecen indicar la presencia de una fase de la Última Glaciación, que se propone llamar Fase Externa de los Canales, cuyas formas deben ser más antiguas que las encontradas recientemente por el autor en los fiordos de la región. A su vez, los rasgos de la zona sin fiordos confluentes debieran indicar la primera etapa de deglaciación en esta fase.

\section{AGRADECIMIENTOS}

El autor expresa su reconocimiento al Comité Oceanográfico Nacional, a su Secretario Ejecutivo Sr. Mario Cáceres y al personal del AGOR Vidal Gormaz participante en el Crucero Campos de Hielo Sur, por haber puesto a su disposición el material analizado.

\section{REFERENCIAS}

Alvarez, W., R. Colacicchi \& A. Montanari. 1985. Synsedimentary slides and bedding formation in Apennine palagic limestones. J. Sedim. Petrol., 55(5): 720-734.

Anderson, J.B. 1993. Antarctic glacial marine sedimentation. In: Bryan, J.R. (ed.). Workshop on Antarctic glacial marine and biogenic sedimentation, notes for a shortcourse. Part 1. Glacial-marine sedimentation. Sedimentology Research Laboratory, Contribution 57, Tallahassee, Florida: 1-88.

Araya-Vergara, J.F. 1997. Perfiles geomorfológicos de los fiordos y depresión longitudinal de Norpatagonia. Cienc. Tecnol. del Mar, 20: 3-22.

Araya-Vergara, J.F. 1998. El problema genético de los fondos de fiordo norpatagónicos. Invest. Mar., Valparaíso, 26: 71-81.

Auton, C.A. 1992. Scottish landform examples. 6. The Flemington Eskers. Scottish Geogr. Mag., 108(3): 190-196.

Barrett, P.J. y M.J. Hambrey, 1992. Plio-Pleistocene sedimentation in Ferrar Fiord, Antarctica. Sedimentology, 39: 109-123.

Brodzikowski, K. 1993. Glacilacustrine sedimentation, Part I: Depositional processes and lithofacies characteristics. Acta Geogr. Lodsziensia, 62. Societas Sci. Lodziensis, Lódz., 136 pp. (En polaco e inglés).

Carlson, P.R. 1989. Seismic reflection characteristics of glacial and glaciomarine sediment in the Gulf. of Alaska and adjacent fjords. Mar. Geol., 85: 391416.

CONA (Comité Oceanográfico Nacional, Chile). 1995. Crucero de investigación científica marina a los fiordos y canales adyacentes a Campos de Hielo Sur: Informe de crucero. CONA, Valparaíso.

Chiocci, F.L., L. Orlando y P. Tortora. 1990. Small scale seismic stratigraphy and paleogeographical evolution of the continental shelf facing the Elba Island, etc. J. Sedim. Petrol., 61(4): 506-526.

Damuth, J.E. 1978. Echo character of the NorwegianGreenland Sea: relationship to Quaternary sedimentation. Mar. Geol., 28: 1-36. 
Domack, E.W. 1990. Climatic and oceanographic controls upon antarctic fjord sedimentation: Examples from the Antarctic Peninsula and South Shetland Islands. Antarctic J. U.S., 35(5): 59-60.

Dowdeswell, J.A., R.J. Whittington y P. Marienfeld. 1994. The origin of massive diamicton facies by iceberg rafting and scouring, Scoresby Sund, East Greenland. Sedimentology, 41: 21-35.

Embley R.W. y M.G. Langseth. 1977. Sedimentation processes on the continental rise of northeastern South America. Mar. Geol., 25: 279-297.

Guilcher, A. 1985. $X X X^{\mathrm{e}}$ Chronique océanographique. Norois, 32 (125): 115-158.

Henderson, P.J. 1988. Sedimentation in an esker system influenced by bedrock topography near Kingston, Ontario. Can. J. Earth Sci., 25: 987-999.

Hervé, F. y Y. Ota. 1993. Fast Holocene uplift rates at the Andes of Chiloé, southern Chile. Rev. Geol. Chile, 20(1): 15-23.

Heusser, C.J. 1990. Chilotan piedmont glacier in the southern Andes during the last glacial maximum. Rev. Geol. Chile, 17: 13-18.

Heusser, C.J. 1997. Deglacial setting of the Southern Andes following the last glacial maximum: a short review. An. Inst. Patagonia, Ser. Cs. Nat. (Chile), 25: 89-103.

Heusser, C.J. y R.F. Flint. 1977. Quaternary glaciations and environments of northern Isla Chiloé, Chile. Geology, 5: 305-308.

Hine, A.C., M.W. Harris, S.D. Locker y P. Hallock. 1994. Sedimentary infilling of an open seaway: Bawihka Channel, Nicaraguan Rise. J. Sedim. Res., 64(1): 2-25.

Josenhans, H.W. y G.B.J. Fader. 1989. A comparison of models of glacial sedimentation along the eastern Canadian margin. Mar. Geol., 85: 273-300.

Karl, H.A. 1989. High-resolution seismic-reflection interpretations of some sediment deposits, Antarctic continental margin: focus of the western Ross Sea. Mar. Geol., 85: 205-223.
Kaszycki, Ch.A. 1987. A model of glacial and proglacial sedimentation in the shield terrane of southern Ontario. Can. J. Earth Sci., 24: 2373-2391.

Lemmen, D.S. 1990. Glaciomarine sedimentation in Disraeli Fiord, High Arctic Canada. Mar. Geol., 94: 9-22.

Leventer, A., S. Aavang, J. Stravers, S. Ishman y L. Krissek. 1995. Holocene sedimentation rates on the southern Chilean continental margin. Antarctic J. of the U.S., 30(5): 13-16.

Mc Cabe, A.M. 1986. Glaciomarine facies deposited by retreating tidewater glaciers: an example from the Late Pleistocene of Northern Ireland. J. Sedim. Petrol., 56(6): 880-894.

Mercer, J. 1970. Variations of some Patagonian glaciers since the Late-Glacial: II. Amer. J. Sci., 269: 1-25.

Powell, R.D. y B.F. Molnia. 1989. Glacimarine sedimentation processes, facies and morphology of the south-southeast Alaska shelf and fjords. Mar. Geol., 85: 359-390.

Pratson, L.F. y E.P. Laine. 1989. The relative importance of gravity-induced versus currentcontrolled sedimentation during the Quaternary along de mideast U.S. outer continental margin revealed by $3.5 \mathrm{kHz}$ echo character. Mar. Geol., 89: 87-126.

Ríos, F. y M. Cisternas. 1998. Uso de parámetros sedimentológicos en el reconocimiento de ambientes hidrodinámicos en los canales y fiordos adyacentes a "Campos de Hielo Sur". Invest. Mar., Valparaíso, 26: 21-30.

Shaw, J. 1971. Mechanism of till deposition related to thermal conditions in a Pleistocene glacier. J. Glaciol., 10: 363-373.

Uchupi, E., S.A. Swift y D.A. Ross. 1996. Gas venting and the late Quaternary sedimentation in the Persian (Arabian) Gulf. Mar. Geol., 129: 237-269.

Young, A. 1975. Slopes. Longman, London, 288 pp. 